\title{
Solid-phase synthesis of 3-hydroxy-2-methylenealkanenitriles using polymer-supported $\alpha$-selenopropionitrile
}

\author{
Qiao-Sheng Hu, ${ }^{a}$ Shou-Ri Sheng, ${ }^{b} *$ Shui-Ping Huang, ${ }^{b}$ Min Lin, ${ }^{b}$ and Ming-Zhong Cai ${ }^{b}$ \\ ${ }^{a}$ College of Chemistry and Life Science, Gannan Normal University, Ganzhou, 341000, \\ P. R. China \\ ${ }^{b}$ College of Chemistry and Chemical Engineering, Jiangxi Normal University, Nanchang, \\ 330027, P. R. China \\ E-mail:shengsr@jxnu.edu.cn
}

\begin{abstract}
A novel facile method for solid-phase organic synthesis of 3-hydroxy-2-methylenealkanenitriles in good yields and purities with advantages of decreased volatility and simplification of work-up procedure using polymer-supported $\alpha$-selenopropionitrile is described.
\end{abstract}

Keywords: Solid-phase organic synthesis, polystyrene-supported $\alpha$-selenopropionitrile, 3-hydroxy-2-methylenealkanenitrile

\section{Introduction}

Polymer-supported organic reagents and catalysts have been rapidly applied in the preparation of organic molecules. The use of polymer-supported reagents can selectively remove excess reagents and by-products through simple filtration rather than liquid-liquid extraction and chromatographic purification. In addition, polymer-supported reagents offer further advantages that include reaction of active intermediates by 'catch-and-release', selectivity and immobilization of toxic intermediates. ${ }^{1}$ Organoselenium reagents have been increasingly used as a powerful tool for introducing new functional groups into organic substrates under extremely mild conditions. ${ }^{2}$ For example, phenylseleno group is readily converted to a leaving group giving access to carbon-carbon double bonds via oxidation followed by $\beta$-elimination. ${ }^{3}$ Additionally, a selenium-stabilized carbanion has played an important role in organic synthesis because of its easy availability and particularly good nucleophile, which allows the formation of new functionalized carbon-carbon bonds when it is used to react with compounds bearing an electrophilic carbon atom. ${ }^{2,4}$ However, organoselenium reagents always have a foul smell and are quite toxic, which is often problematic in organic synthesis. Although polymers with selenium functionalities have been known for along time, ${ }^{5}$ there remains high interest in this kind 
of solid-phase organic chemistry. ${ }^{6}$ The Baylis-Hillman reaction has become an important tool in organic synthesis, since it allows for the formation of carbon-carbon bonds under mild reaction conditions. $^{7} \quad$ The adducts (3-hydroxy-2-methylenealkanenitriles and 3-hydroxy-2methylenealkanoates, etc.) of Baylis-Hillman reaction have recently been utilized1 as important precursors for stereoselective synthesis of different multifunctional molecules. ${ }^{8}$ Although methods for the synthesis of 3-hydroxy-2-methylenealkanenitriles and their derivatives are well documented, 7,9 efforts are continuing for the development of more efficient methods with experimental simplicity. However, to our knowledge, solid-phase organic synthesis (SPOS) of 3hydroxy-2-methylenealkanenitriles using selenium linkage has not been reported. In connection with our interest in solid-phase organoselenium chemistry, ${ }^{10}$ we wish herein to report the preparation of the novel polymer-bound $\alpha$-selenopropionitrile reagent and its application for the SPOS of 3-hydroxy-2-methylenealkanenitriles (Scheme 1).



1

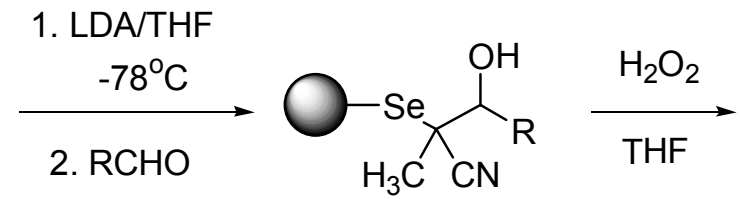

4

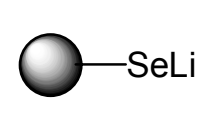

2



3



5

6

Scheme 1. SPOS of 3-hydroxy-2-methylenealkanenitriles.

\section{Results and Discussions}

As shown in Scheme 1, polymer-supported $\alpha$-selenopropionitrile 3 were readily prepared by treatment of a THF-swollen suspension of cross-linked (1\%) polystyrene-bound selenium bromide $1^{6 \mathrm{a}}$ with $\mathrm{LiBH}_{4}$, followed by treatment with 2-bromopropionitrile. Elemental analysis for nitrogen revealed a loading of resin $3(1.20 \mathrm{mmol} / \mathrm{g})$. No bromine was found by elemental analysis, indicating that the performed bromine with $\alpha$-propionitrile group exchange has gone to completion. The FT-IR spectrum of the newly formed resin 3 showed a strong stretching vibration of the cyano group at $2241 \mathrm{~cm}^{-1}$. A distinct advantage of the novel polymer reagents is the convenience of handling and odorless nature as compared with the nonpolymer-supported reagents.

With resin 3 in hand, preparation of 3-hydroxy-2-methylenealkanenitriles based on resin $\mathbf{3}$ was investigated. As illustrated, reaction of the lithio derivative of resin $\mathbf{3}$ generated by treating resin 3 with lithium diisopropylamide with aldehyde furnished the resin $\mathbf{4}$, which could not be 
reliably analyzed with FT-IR. Hence next step of oxidation-elimination reaction was carried out directly with $30 \%$ hydrogen peroxide at $0{ }^{\circ} \mathrm{C}$ and then at room temperature to afford the corresponding Baylis-Hillman products 5 in good yields (82-91\%) and purities (92-96 \%). Several typical examples are described in Table 1. Seen from Table 1, the present method was effective for both aromatic (either with electron-withdrawing or electron-donating group) and aliphatic aldehydes.

Table 1. The yields and purities of 3-hydroxy-2-methylenealkanenitriles 5

\begin{tabular}{ccccc}
\hline Entry & $\mathrm{R}$ & Product & Yield (\%) $^{a}$ & ${\text { Purity }(\%)^{b}}^{b}$ \\
\hline $\mathbf{1}$ & $\mathrm{C}_{6} \mathrm{H}_{5}$ & $\mathbf{5 a}$ & 90 & 95 \\
$\mathbf{2}$ & $4-\mathrm{CH}_{3} \mathrm{OC}_{6} \mathrm{H}_{4}$ & $\mathbf{5 b}$ & 91 & 96 \\
$\mathbf{3}$ & $4-\mathrm{ClC}_{6} \mathrm{H}_{4}$ & $\mathbf{5 c}$ & 88 & 95 \\
$\mathbf{4}$ & $4-\mathrm{NO}_{2} \mathrm{C}_{6} \mathrm{H}_{4}$ & $\mathbf{5 d}$ & 85 & 92 \\
$\mathbf{5}$ & 2-naphthyl & $\mathbf{5 e}$ & 84 & 95 \\
$\mathbf{6}$ & 2-furyl & $\mathbf{5 f}$ & 86 & 93 \\
$\mathbf{7}$ & 3-pyridyl & $\mathbf{5 g}$ & 87 & 95 \\
$\mathbf{8}$ & $n-\mathrm{C}_{5} \mathrm{H}_{11}$ & $\mathbf{5 h}$ & 82 & 92 \\
\hline
\end{tabular}

${ }^{a}$ Yields were based on the functional loading of resin $3(1.20 \mathrm{mmol} / \mathrm{g}) .{ }^{b}$ Determined by HPLC of crude cleavage product.

The residual resin, polystyrene-supported phenylseleninic acid $\mathbf{6}$, was obtained as a byproduct, whose infrared data was identical to the previously reported data ${ }^{11}$ and showed no residual cyano group absorption, which indicated the oxidative cleavage was complete. The resin $\mathbf{6}$ could be converted to selenium bromide resin $\mathbf{1}$ for recycle by treatment of it with $\mathrm{KI} / \mathrm{Na}_{2} \mathrm{~S}_{2} \mathrm{O}_{3}$ 12 followed by bromine. ${ }^{6 a}$ To show the reactivity of the regenerated polymeric reagent, the conversion of polymeric selenium bromide $\mathbf{1}$ to target compounds 5 was repeated several times in the same reaction. For instance, 3-hydroxy-2-methylene-3-phenylpropanenitrile (5a) was obtained in $87 \%$ yield and with $92 \%$ purity under the same reaction condition using the selenenyl bromide resin 1 (second run), and in $83 \%$ yield after second recycle (i.e. third run). It was shown that recycling 2-3 times led to a gradual decrease in yield but the purity of 5a remained almost the same as when the first prepared selenium bromide resin was used.

In summary, an efficient and convenient method for the solid-phase synthesis of 3-hydroxy-2methylenealkanenitriles in good yields and purities employing polymer-bound $\alpha$ selenopropionitrile reagent has been developed. The advantages of this method include straightforward operation, lack of odor and stability, and the polymeric reagent can be regenerated and reused for several times as environmentally benign reagent. 


\section{Experimental Section}

General Procedure. Melting points were uncorrected. ${ }^{1} \mathrm{H}$ NMR (400 MHz) and ${ }^{13} \mathrm{C}$ NMR (100 $\mathrm{MHz}$ ) spectra were recorded on a Bruker Avance (400 MHz) spectrometer, using $\mathrm{CDCl}_{3}$ as the solvent and TMS as internal standard. FT-IR spectra were taken on a Perkin-Elmer SP One FT-IR spectrophotometer. Microanalyses were performed with a Carlo Erba 1106 Elemental Analyzer. HPLC analysis was performed on Agilent 1100 automated system having a PDA detector $\left(\lambda_{\max }=\right.$ $254 \mathrm{~nm}$ used for this study) using a gradient with $\mathrm{CH}_{3} \mathrm{CN} / \mathrm{H}_{2} \mathrm{O}(1 \mathrm{~mL} / \mathrm{min})$ on a RP-18e column (150×4.6 mm). Polystyrene (H 1000, 100-200 mesh, cross-linked with 1\% divinylbenzene) for preparation of selenium bromide resin ${ }^{6 a}$ was purchased from Nankai University, and the other starting materials were purchased from commercial suppliers and used without further purification. THF was stilled from sodium-benzophenone immediately prior to use.

\section{Preparation of polymer-supported selenium bromide 1}

Under a positive pressure of nitrogen, to $1 \%$ divinylbenzene-styrene copolymer $(2.0 \mathrm{~g}, 19.3$ mequiv) swollen in dry cyclohexane $(20 \mathrm{~mL})$ containing $2.8 \mathrm{~mL}(18.6 \mathrm{mmol})$ of tetramethylethylenediamine (TMEDA) was added $9.5 \mathrm{ml}$ of $2.50 \mathrm{M} \mathrm{n}$-BuLi. The reaction mixture turned red gradually during $4.5 \mathrm{~h}$ of heating at $65{ }^{\circ} \mathrm{C}$. After the liquid phase was removed, the resin was rinsed twice with dry cyclohexane to yield the desired lithiated resin. Then the lithiated resin was quenched by addition of a solution of dimethyl diselenide (5.0 mmol) in dry tetrahydrofuran $(10 \mathrm{~mL})$ with stirring at $0{ }^{\circ} \mathrm{C}$ for $30 \mathrm{~min}$. The brown coloration of the resin disappeared instantly. After filtration, the resin was washed with THF, methanol, THFwater (2:1), water, THF, and finally methanol (10 mL of each). After drying in vacuo at $60{ }^{\circ} \mathrm{C}$ for $10 \mathrm{~h}$, the polymer-supported methyl selenide $(2.12 \mathrm{~g})$ as a pale yellow resin containing 1.28 mmol Se/g was obtained. The methyl selenide resin $(2.0 \mathrm{~g})$ swollen in $\mathrm{CHCl}_{3}(10 \mathrm{~mL})$ was added $\mathrm{Br}_{2}(0.37 \mathrm{~g}, 2.3 \mathrm{mmol})$ and the mixture was stirred at $0{ }^{\circ} \mathrm{C}$ for $10 \mathrm{~min}$. After filtration, the resin was washed twice with $\mathrm{CHCl}_{3}(2 \times 10 \mathrm{~mL})$ and then was suspended in dry ethanol $(15 \mathrm{~mL})$. After stirring at $70{ }^{\circ} \mathrm{C}$ for $1 \mathrm{~h}$, the resin was collected on a filter and washed successively with THF, ether and finally methanol (10 mL of each). After drying, $2.05 \mathrm{~g}$ of a pale yellow polystyrenesupported selenium bromide resin $(1.18 \mathrm{mmol} \mathrm{Br} / \mathrm{g}$, the loading of functional $\mathrm{Br}$ was analyzed by elementary analysis) was obtained. IR (KBr): $v=3024,2920,1600,1492,1452,1028,757,698$, $540 \mathrm{~cm}^{-1}$.

\section{Preparation of polymer-supported $\alpha$-selenopropionitrile 3}

Under a nitrogen atmosphere, to polystyrene-supported selenium bromide 1 (1.0 g, $1.18 \mathrm{mmol})$ swelled in THF $(10 \mathrm{~mL})$ for $30 \mathrm{~min}$ was added $\mathrm{LiBH}_{4}(3 \mathrm{mmol})$. After $1 \mathrm{~h}$ with shaking at room temperature, 2-bromopropionitrile $(3 \mathrm{mmol})$ in $2 \mathrm{~mL}$ of THF was added slowly and the mixture was shaken for $6 \mathrm{~h}$. The resin was collected on a filter and washed successively with $\mathrm{H}_{2} \mathrm{O}(2 \times 20$ $\mathrm{mL})$, THF $(3 \times 5 \mathrm{~mL})$ and $\mathrm{CH}_{2} \mathrm{Cl}_{2}(3 \times 5 \mathrm{~mL})$, and then dried under vacuum overnight to afford 930 $\mathrm{mg}$ of resin 3 in $96 \%$ yield with a loading value of $1.20 \mathrm{mmol} / \mathrm{g}$ (theoretical loading of the resin 
$1.22 \mathrm{mmol} / \mathrm{g}) . \mathrm{IR}(\mathrm{KBr}): v=3025,2926,2241,1596,1460,1405,1378,1304,1095,1024,999$, $740,690,672 \mathrm{~cm}^{-1}$.

\section{General procedure for the preparation of 3-hydroxy-2-methylenealkanenitriles (5a-5h)}

To a solution of $2.0 \mathrm{mmol}$ of LDA (2.2 mmol of diisopropylamine, $2.0 \mathrm{mmol}$ of n-butyllithium) in anhydrous THF $(10 \mathrm{~mL})$ at $-78{ }^{\circ} \mathrm{C}$ under nitrogen atmosphere was added resin 3 (833 $\mathrm{mg}, 1.0$ mmol). After shaking at $-78{ }^{\circ} \mathrm{C}$ for $1 \mathrm{~h}$, the neat aldehyde $(2.0 \mathrm{mmol}$ in $5 \mathrm{~mL}$ THF $)$ was added dropwise over ca. $5 \mathrm{~min}$. Twenty minutes after addition of the aldehyde, the mixture was warmed up gradually to room temperature and kept shaking for $3 \mathrm{~h}$. After neutralization with $1 \%$ hydrochloric acid, the resin $\mathbf{4 a - 4 h}$ was collected on a filter and washed successively with $\mathrm{H}_{2} \mathrm{O}$ (3 $\times 10 \mathrm{~mL})$, THF $(3 \times 5 \mathrm{~mL})$ and $\mathrm{Et}_{2} \mathrm{O}(3 \times 5 \mathrm{~mL})$. To a suspension of the swollen resin $\mathbf{4 a - 4 h}$ in THF $(10 \mathrm{~mL})$ and $0.5 \mathrm{~mL}$ of $30 \% \mathrm{H}_{2} \mathrm{O}_{2}(5.8 \mathrm{mmol})$ was added at $0{ }^{\circ} \mathrm{C}$. The suspension was shaken at $0{ }^{\circ} \mathrm{C}$ for $0.5 \mathrm{~h}$ and then at room temperature for $1 \mathrm{~h}$, the residual resin was collected by filtration and washed with $\mathrm{CH}_{2} \mathrm{Cl}_{2}(3 \times 10 \mathrm{~mL})$. The filtrate was treated with saturated $\mathrm{NaHCO}_{3}$ $(20 \mathrm{~mL})$ and washed with water, dried over magnesium sulfate and evaporated to give crude products $\mathbf{5 a - 5 h}$, which were further purified by chromatography [silica gel, ethyl acetate/hexane as the eluent: $1: 9-1.5: 8.5(\mathrm{v} / \mathrm{v})]$ to provide pure products for their structure analyses.

3-Hydroxy-2-methylene-3-phenylpropanenitrile (5a). Colorless oil (lit. ${ }^{9 a}$ oil). ${ }^{1} \mathrm{H}$ NMR: $\delta=7.41-7.35(\mathrm{~m}, 5 \mathrm{H}), 6.11(\mathrm{~s}, 1 \mathrm{H}), 6.03(\mathrm{~s}, 1 \mathrm{H}), 5.28(\mathrm{~s}, 1 \mathrm{H}), 2.58$ (br s, $1 \mathrm{H}) .{ }^{13} \mathrm{C}$ NMR: $\delta=141.3,130.9,128.7,127.9,127.5,123.8,118.6,72.2$. IR (neat): $v=3345,1630,2225,1250$ $\mathrm{cm}^{-1}$. Anal. Calcd for $\mathrm{C}_{10} \mathrm{H}_{9} \mathrm{NO}$ : C, 75.45; H, 5.70; N, 8.80. Found: C, 75.53; H, 5.82; N, 8.89.

3-Hydroxy-2-methylene-3-(4-methoxyphenyl)propanenitrile (5b). Yellow oil (lit. ${ }^{\text {a }}$ yellow oil). ${ }^{1} \mathrm{H}$ NMR: $\delta=7.46$ (d, $\left.J=8.5 \mathrm{~Hz}, 2 \mathrm{H}\right), 6.76(\mathrm{~d}, J=8.5 \mathrm{~Hz}, 2 \mathrm{H}), 6.34(\mathrm{~s}, 1 \mathrm{H}), 6.05$ (s, $\left.1 \mathrm{H}\right)$, $4.74(\mathrm{~s}, 1 \mathrm{H}), 3.74$ (s, $3 \mathrm{H}), 2.16$ (br s, $1 \mathrm{H}) .{ }^{13} \mathrm{C} \mathrm{NMR}: \delta=131.2,130.1,123.6,120.2,118.9$, 114.1, 113.5, 72.2, 55.2. IR (neat): $v=3284,2860,2226,1622,1454,1386,1248 \mathrm{~cm}^{-1}$. Anal. Calcd for $\mathrm{C}_{11} \mathrm{H}_{11} \mathrm{NO}_{2}$ : C, 69.83; H, 5.86; N, 7.40. Found: C, 69.90; H, 5.95; N, 7.49.

3-Hydroxy-2-methylene-3-(4-chlorophenyl)propanenitrile (5c). Yellow oil (lit. ${ }^{13}$ yellow oil). ${ }^{1} \mathrm{H}$ NMR: $\delta=7.43(\mathrm{~d}, J=8.6 \mathrm{~Hz}, 2 \mathrm{H}), 7.28$ (d, $\left.J=8.6 \mathrm{~Hz}, 2 \mathrm{H}\right), 6.09$ (s, $\left.1 \mathrm{H}\right), 6.05$ (s, $\left.1 \mathrm{H}\right)$, 5.26 (s, $1 \mathrm{H}), 2.32$ (br s, $1 \mathrm{H}) .{ }^{13} \mathrm{C} \mathrm{NMR}: \delta=140.3,133.5,130.8,128.4,127.9,123.8,119.1$, 72.3. IR (neat): $v=3308,2224,1625,1406 \mathrm{~cm}^{-1}$. Anal. Calcd for $\mathrm{C}_{10} \mathrm{H}_{8} \mathrm{ClNO}$ : C, 62.03; H, 4.16; N, 7.23. Found: C, 62.13; H, 4.24; N, 7.32.

3-Hydroxy-2-methylene-3-(4-nitrophenyl)propanenitrile (5d). Pale yellow solid; Mp 73-74 ${ }^{\circ} \mathrm{C}$ (lit. $\left.{ }^{13} \mathrm{mp} 72-75{ }^{\circ} \mathrm{C}\right) .{ }^{1} \mathrm{H}$ NMR: $\delta=8.25(\mathrm{~d}, J=8.6 \mathrm{~Hz}, 2 \mathrm{H}), 7.62(\mathrm{~d}, J=8.6 \mathrm{~Hz}, 2 \mathrm{H}), 6.19$ (s, $1 \mathrm{H}), 6.09$ (s, $1 \mathrm{H}), 5.42$ (s, $1 \mathrm{H}), 2.12$ (br s, $1 \mathrm{H}) .{ }^{13} \mathrm{C} \mathrm{NMR}: \delta=147.9,147.2,130.7,127.2$, 126.1, 123.3, 118.6, 72.1. IR (KBr): $v=3440,2227,1618,1552 \mathrm{~cm}^{-1}$. Anal. Calcd for $\mathrm{C}_{10} \mathrm{H}_{8} \mathrm{~N}_{2} \mathrm{O}_{3}$ : C, 58.82; H, 3.95; N, 13.72. Found: C, 58.95; H, 4.04; N, 13.56.

3-Hydroxy-2-methylene-3-(2-naphthyl)propanenitrile (5e). Yellow oil (lit. ${ }^{9 a}$ yellow oil). ${ }^{1} \mathrm{H}$ NMR: $\delta=7.92-7.34(\mathrm{~m}, 7 \mathrm{H}), 6.16(\mathrm{~s}, 1 \mathrm{H}), 5.85(\mathrm{~s}, 1 \mathrm{H}), 4.82(\mathrm{~s}, 1 \mathrm{H}), 2.35$ (br s, $1 \mathrm{H}) .{ }^{13} \mathrm{C}$ NMR: $\delta=138.7,133.4,132.7,130.9,128.5,128.1,127.6,127.1,125.7,125.4,124.2,123.4$, 119.2, 72.3. IR (neat): $v=3435,2221,1623 \mathrm{~cm}^{-1}$. Anal. Calcd for $\mathrm{C}_{14} \mathrm{H}_{11} \mathrm{NO}$ : C, 80.36; H, 5.30; 
N, 6.69. Found: C, 80.41; H, 5.44; N, 6.76.

3-Hydroxy-2-methylene-3-(2-furyl)propanenitrile (5f). Organe oil (lit. ${ }^{14}$ oil). ${ }^{1} \mathrm{H}$ NMR: $\delta=7.45(\mathrm{dd}, J=1.7,0.9 \mathrm{~Hz}, 1 \mathrm{H}), 6.45(\mathrm{dd}, J=3.2,1.7 \mathrm{~Hz}, 1 \mathrm{H}), 6.40(\mathrm{dd}, J=3.2,0.9 \mathrm{~Hz}, 1 \mathrm{H})$, $6.21(\mathrm{~s}, 1 \mathrm{H}), 6.16(\mathrm{~s}, 1 \mathrm{H}), 5.38(\mathrm{~s}, 1 \mathrm{H}), 2.45$ (br s, $1 \mathrm{H}) .{ }^{13} \mathrm{C}$ NMR: $\delta=151.4,143.5,131.1$, 123.6, 116.7, 110.7, 108.9, 67.7. IR (neat): $v=3418,2229,1625,1503 \mathrm{~cm}^{-1}$. Anal. Calcd for $\mathrm{C}_{8} \mathrm{H}_{7} \mathrm{NO}_{2}: \mathrm{C}, 64.42 ; \mathrm{H}, 4.73 ; \mathrm{N}, 9.39$. Found: C, 64.50; H, 4.81; N, 9.46.

3-Hydroxy-2-methylene-3-(3-pyridyl)propanenitrile (5g). Colorless oil. (lit. ${ }^{9 a}$ oil). ${ }^{1} \mathrm{H}$ NMR: $\delta=8.65(\mathrm{~s}, 1 \mathrm{H}), 8.50(\mathrm{~d}, J=4.9 \mathrm{~Hz}, 1 \mathrm{H}), 7.70(\mathrm{~d}, J=8.0 \mathrm{~Hz}, 1 \mathrm{H}), 7.14(\mathrm{dd}, J=4.9,8.0 \mathrm{~Hz}, 1$ $\mathrm{H}), 6.06$ (s, $1 \mathrm{H}), 5.84(\mathrm{~s}, 1 \mathrm{H}), 4.75$ (s, $1 \mathrm{H}), 5.15$ (br s, $1 \mathrm{H}) .{ }^{13} \mathrm{C} \mathrm{NMR}: \delta=148.5,148.2,138.3$, 135.1, 130.6, 124.1, 123.5, 117.8, 70.4. IR (neat): $v=3430,2219,1623,1500 \mathrm{~cm}^{-1}$. Anal. Calcd for $\mathrm{C}_{9} \mathrm{H}_{8} \mathrm{~N}_{2} \mathrm{O}$ : C, 67.49; H, 5.03; N, 17.49. Found: C, 67.60; H, 5.12; N, 17.56.

3-Hydroxy-2-methyleneoctanenitrile (5h). Colorless oil (lit. ${ }^{8 \mathrm{c}}$ oil). ${ }^{1} \mathrm{H}$ NMR: $\delta=6.24$ (s, $1 \mathrm{H}$ ), $5.83(\mathrm{~s}, 1 \mathrm{H}), 4.43$ (t, $J=6.4 \mathrm{~Hz}, 1 \mathrm{H}), 4.27$ (q, $J=6.4 \mathrm{~Hz}, 2 \mathrm{H}), 2.74$ (br s, 1H), 1.41-1.26 (m, 6 $\mathrm{H}), 0.97$ (t, $J=7.1 \mathrm{~Hz}, 3 \mathrm{H}) .{ }^{13} \mathrm{C}$ NMR: $\delta=134.1,123.9,118.6,70.0,33.6,29.6,23.5,19.9$, 12.2. IR (film): $v=3380,2958,2862,2214,1625 \mathrm{~cm}^{-1}$. Anal. Calcd for $\mathrm{C}_{9} \mathrm{H}_{15} \mathrm{NO}: \mathrm{C}, 70.55 ; \mathrm{H}$, 9.87; N, 9.14. Found: C, 70.62; H, 9.98; N, 9.22.

\section{Acknowledgements}

We gratefully acknowledge financial support from the National Natural Science Foundation of China (No. 20562005), NSF of Jiangxi Province (No. 0620021 and No. 2007GZW0185) and the Research Program of Jiangxi Province Department of Education (No. GJJ08165).

\section{References}

1. For recent reviews, see: (a) Shuttleworth, S. J.; Allin, S. M.; Sharma, P. K. Synthesis 1997, 1217. (b) Guillier, F.; Orain, D.; Bradley, M. Chem. Rev. 2000, 100, 2091. (c) Sammelson, R. E.; Kurth, M. J. Chem. Rev. 2001, 101, 137. (d) Zhdankin, V. V.; Stang, P. J. Chem. Rev. 2002, 102, 2523.

2. (a) Paulmier, C.; Ed. Selenium Reagents and Intermediates in Organic Synthesis, Pergamon Press: Oxford, 1986. (e) Liotta, D.; Ed. Organoselenium Chemistry, Wiley: New York, 1987. (f) Krief, A. Comprehensive Organic Synthesis, Pergamon: Oxford, 1991. (g) Back, T. G. Organoselenium Chemistry, Oxford University Press: Oxford, 1999; pp 173-191. (h) Wirth, T. Organoselenium Chemistry, Springer: Berlin, 2000.

3. Reich, H. J. Acc. Chem. Res. 1979, 12, 22.

4. (a) Krief, A. Tetrahedron 1980, 36, 2531. (b) Rollinson, S. W.; Amos, R. A.; Katzenellenbogen, J. A. J. Am. Chem. Soc. 1981, 103, 4114.

5. Michels, R.; Kato, M.; Heitz, W. Makromol. Chem. 1976, 177, 2311. 
6. (a) Nicolaou, K. C.; Pastor, J.; Barluenga, S.; Winssinger, N. Chem. Commun. 1998, 1947.

(b) Ruhland, T.; Andersen, K.; Pedersen, H. J. Org. Chem. 1998, 63, 9204. (c) Yanada, K.; Fujita, T.; Yanada, R. Synlett 1998, 971. (d) Zaragoza, F. Angew. Chem. Int. Ed. 2000, 39, 2077. (e) Li, Z.; Kulkarni, B. A.; Ganesan, A. Biotechnol. Bioeng. 2001, 71, 104. (f) Uehlin, L.; Wirth, T. Org. Lett. 2001, 3, 2931. (g) Fujita, K.-I.; Hashimoto, S.; Oishi, A.; Taguchi, Y. Tetrahedron Lett. 2003, 44, 3793. (h) Berlin, S.; Ericsson, C.; Engman, L. J. Org. Chem. 2003, 68, 8386. (i) Mogemark, M.; Gustafsson, L.; Bengtsson, C.; Elofsson, M.; Kihlberg, J. Org. Lett. 2004, 6, 4885. (j) Cohen, R. J.; Fox, D. L.; Salvatore, R. N. J. Org. Chem. 2004, 69, 4265. (k) Barrero, A. F.; Quílez del Moral, J. F.; Herrador, M. M.; Herrador, M. M.; Cortés, M.; Arteaga, P.; Catalán, J. V.; Sánchez, E. M.; Arteaga, J. F. J. Org. Chem. 2006, $71,5811$.

7. For review, see: (a) Drewes, S. E.; Roos, G. H. P. Tetrahedron 1988, 44, 4653. (b) Basavaiah, D.; Rao, P. D.; Hyma, R. S. Tetrahedron 1996, 52, 8001. (c) Ciganek, E. Org. React. 1997, 51, 201. (d) Marko, I. E.; Giles, G. P.; Hindley, N. J. Tetrahedron 1997, 53, 1015. (e) Langer, P. Angew. Chem., Int. Ed. Engl. 2000, 39, 3049. (f) Basavaiah, D.; Rao, A. J.; Satyanarayana, T. Chem. Rev. 2003, 103, 811.

8. (a) Hoffmann, H. M. R.; Rabe, J. Angew. Chem., Int. Ed. 1985, 24, 94. (b) Buchholz, R.; Hoffmann, H. M. R. Helv. Chim. Acta 1991, 74, 1213. (c) Basavaiah, D.; Bakthadoss, M.; Pandiaraju, S. J. Chem. Soc., Chem. Commun. 1998, 1639.

9. For some recent examples for preparation of 3-hydroxy-2-methylenealkanenitriles, see: (a) de Souza, R. O. M. A.; Meireles, B. A.; Aguiar, L. C. S.; Vasconcellos, M. L. A. A. Synthesis 2004, 1595. (b) Krishna, P. R.; Sekhar, E. R.; Kannan, S. V. Synthesis 2004, 857. (c) Krishna, P. R.; Lopinti, K. R.; Kannan, V. Tetrahedron Lett. 2004, 45, 7847. (d) Krishna, P. R.; Manjuvani, A.; Kannan, V.; Sharma, G. V. M. Tetrahedron Lett. 2004, 45, 1183. (e) Mi, X. L.; Luo, S. Z.; Cheng, J.-P. J. Org. Chem. 2005, 70, 2338. (f) Caumul, P.; Hailes, H. C. Tetrahedron Lett. 2005, 46, 8125. (g) Krishna, P. R.; Manjuvani, A.; Sekhar, E. P. ARKIVOC 2005, (iii), 99.

10. (a) Huang, X.; Sheng S.-R. Tetrahedron Lett. 2001, 42, 9035. (b) Huang, X.; Sheng S.-R. J. Comb. Chem. 2003, 5, 805. (c) Sheng, S.-R.; Liu, X.-L.; Wang, X.-C.; Xin, Q.; Song, C.-S. Synthesis 2004, 2833. (d) Sheng, S.-R.; Xin, Q.; Liu, X.-L.; Sun, W.-K.; Guo, R.; Huang, X. Synthesis 2006, 2293. (e) Sheng, S.-R.; Huang, F.-F.; Lin, S.-Y.; Liu, X.-L.; Huang, X. Synthesis 2007, 1373. (f) Fu, G.-Y.; Guo, L.; Mao, X.-C.; Sheng, S.-R.; Fei, S.-Y.; Cai, M.Z., Arkivoc 2008, 287.

11. Zundel, G. Angew. Chem., Int. Ed. 1969, 8, 499.

12. (a) Ferranti, F.; Filippo, D. D. J. Chem. Soc. (B). 1971, 1925. (b) Huang, X.; Xu, W.-M. Tetrahedron Lett. 2002, 43, 5495.

13. Cai, J.; Zhou, Z.; Zhao, G.; Tang, C. Org. Lett. 2002, 4, 4723.

14. Aggarwal, V. K.; Emme, I.; Fulford, S. Y. J. Org. Chem. 2003, 68, 692. 